\title{
Unusual Vascular Access for Extracorporeal Membrane Oxygenation Implantation a Case Report
}

\author{
Marisol G Ascencio-Lemus ${ }^{1,3 *}$, Pasquale Maiorano², Laura Castillo Pardo ${ }^{2}$ and Mario Castaño Ruiz ${ }^{2}$
}

\author{
${ }^{1}$ Department of interventional cardiology, Hospital Universitario Virgen de Macarena, Spain \\ ${ }^{2}$ Department of Cardiacsurgery, Complejo Asistencial Universitario de León, Spain \\ ${ }^{3}$ Department of cardiology, Complejo Asistencial Universitario de Leon, Spain
}

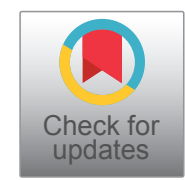

\section{Introduction}

The venoarterial extracorporeal membrane oxygenation ( $V$-A ECMO) has emerged as a life-saving measure for patients in cardiac-respiratory failure in order to provide a mechanical support when conventional strategies have failed, allowing time for recovery, decision-making, or bridging to implantation of a long-term mechanical circulatory support device and occasionally heart transplantation [1].

In an emergency scenario, peripheral cannulation is the usual approach since it can be achieved rapidly and a sternotomy is avoided. The femoral artery is the standard site for vascular access when placing a patient in VA ECMO. Nevertheless, in patients with severe peripheral arterial disease or aneurysm, alternative accesses are the subclavian and the axillary arteries [2,3]. We present a case of a patient with severe peripheral arterial disease in a refractory cardiogenic shock after a complication from $\mathrm{PCl}$ who was deemed for ECMO implantation.

\section{Case Report}

A 60-year-old man who was admitted to the cardiology department due to recurrent syncope and a positive stress echocardiography. Medical background includes dyslipidemia, cerebrovascular disease: Right internal carotid (endarterectomy, 1998), severe left internal carotid stenosis, medical management. Peripheral vascular disease (bi-iliac stent in 2001, aortic bifemoral bypass in 2010) and coronary artery disease, treated by CABG in 2006 (LIMA to LAD. RIMA to OM [T-graft to LIMA]. RA to PDA. Three months prior to admission, he began to experience loss of consciousness preceded with nausea, non-related to efforts, and no chest pain, being admitted for studies.

No relevant data at physical examination was found. ECG at admission was a sinus rhythm with $T$ wave inversion in leads I, AVL, V1-V2, previously present. Cardiac holter revealed no arrhythmias. An angio-CT was requested, reporting a moderate stenosis in right carotid territory, a total occlusion of left internal and common carotid arteries and moderate stenosis in both subclavian arteries. Basal echocardiogram reported a LVEF of 55\%, inferior akinesis and apical hypokinesis. During his hospitalization, ECG exhibited new
T-wave inversion in leads V2-V6. A stress echocardiography confirmed the presence of ischemia in the LAD territory; therefore he underwent an angiography that evidenced severe stenosis in the LAD immediately distal to the anastomosis of the LIMA graft, being permeable all of the grafts and no new stenosis in the other native coronary arteries (Figure 1). Thus, it was decided to perform a PCl on the LAD through the LIMA graft. After guide wire introduction to distal LAD, the patient experienced hemodynamic instability with severe hypotension and bradycardia and then cardiac arrest.

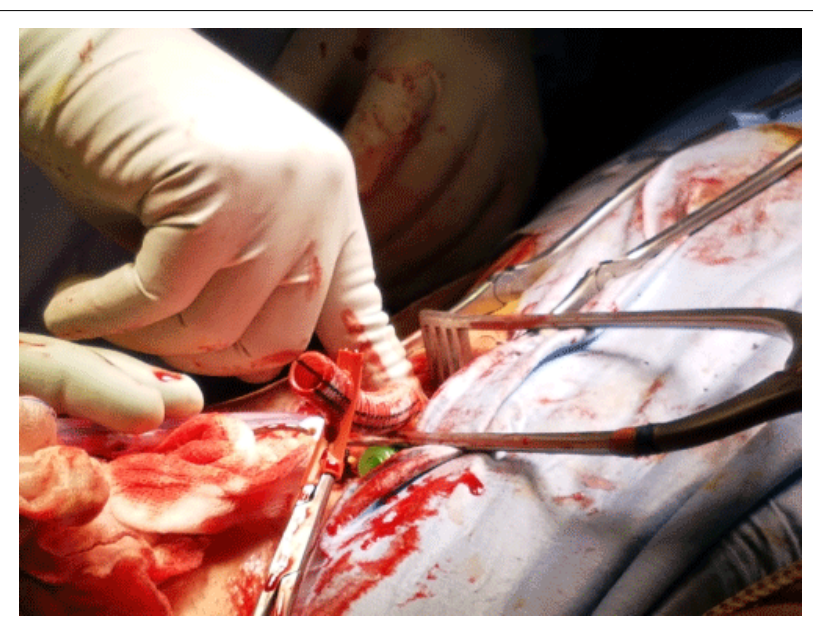

Figure 1: The anastomosis of the LIMA graft.

*Corresponding author: Marisol G Ascencio-Lemus, Department of interventional cardiology, Hospital Universitario Virgen de Macarena, Avenida Dr Fedriani, 3, 41009. Seville, Spain

Accepted: December 23, 2021

Published online: December 27, 2021

Citation: Ascencio-Lemus MG, Maiorano P, Pardo LC, et al. (2021) Unusual Vascular Access for Extracorporeal Membrane Oxygenation Implantation a Case Report. J Cardiothorac Surg Ther 5(1):114-115 

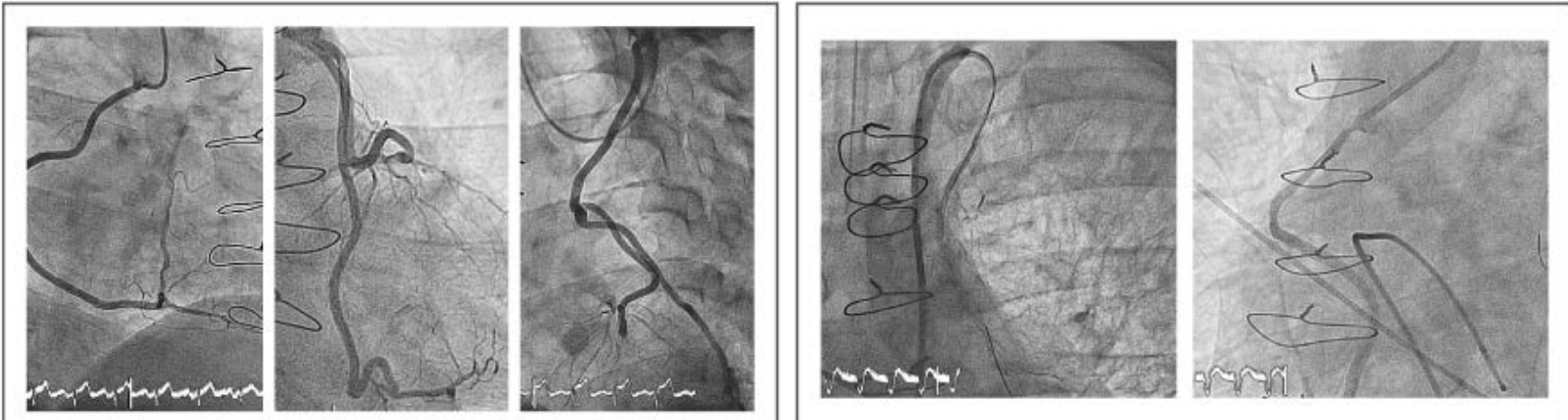

Figure 2: Placing an end-to-side Dacron graft sewn into the right branch of the previous Dacron graft to insert the arterial ECMO cannula.

Advanced life support was initiated, including orotracheal intubation and pacemaker implantation obtaining a pulse recovery in less than 10 minutes, high doses of amines were required. The angiogram exhibited severe spasm of the RA graft and loss of caliber in the proximal segment of LIMA, hence it was performed a DES implantation $3 / 48 \mathrm{~mm}$ into the LIMA graft, with a flow recovery after administration of intracoronary nitroglycerin. An echocardiogram at the bedside of the patient revealed a LVEF of $10 \%$, no pericardial effusion, and due to the presence of refractory cardiogenic shock, the patient was tended for ECMO placing plus intraaortic balloon pump, as a bridge to recovery.

Given the severe atherosclerotic disease, stenosis in the carotid and subclavian arteries and the presence of the aortic bifemoral graft, the artery cannulation was a total challenge. It was planned an approach through the previous aortofemoral graft: by placing an end-to-side Dacron graft sewn into the right branch of the previous Dacron graft to insert the arterial ECMO cannula (Figure 2). Percutaneous approach was tried for venous cannulation, but it was unsuccessful due to the presence of left superficial femoral hematoma. Thus, it was achieved by open dissection. Adequate cannula position at the right atrium was confirmed by transoesophageal echocardiography.

During his stay at the Critical Care Unit, sufficient flow, adequate oxygenation and ventricular unloading were proved. Neurological assessment after arousal from sedation confirmed integrity in neurological status. Sinus rhythm was recovered from the first day, thus, the transient pacemaker was removed. As complications, the patient required blood transfusion due continuous small bleeding at puncture sites during the first day.

Then, hemodynamic stability with an improvement in clinical condition and LVEF (median BP $65 \mathrm{mmHg}$, LVEF $35 \%$,) allowed weaning from ECMO and decannulation was successfully achieved on the $5^{\text {th }}$ postoperative day. A minor swelling on the right leg with no evidence of ischemia was reported as a local complication.

\section{Discussion}

In this case, we have a 60-year-old patient, with an acute myocardial injury and in-hospital cardiac arrest with adequate advanced life support since the very beginning, with pulse recovery in less than 10 minutes and hemodynamic instability refractory to medical treatment. Considering all these factors, heart team decided that our patient was a candidate for short mechanical support with ECMO (as a bridge to an eventual recovery) despite of severe peripheral vascular disease, which is considered in several guidelines a relative contraindication for ECMO implantation.

Arterial cannulation was a total surgical challenge considering the urgency of the procedure. We decide to place an end-to-side Dacron graft sewn to the right branch of the previous Dacron graft for the insertion of the arterial cannula. This one was positioned at the highest level allowed by the cannula and the grafts features, having a sufficient progression into the graft, until graft bifurcation, to optimize and improve retrograde arterial flow and guarantee an adequate brain and heart oxygenation. In addition, it was decided to place an intra-aortic balloon pump as an adjuvant therapy to ECMO, given the better outcomes showed in previous studies [4].

The position of the arterial cannula in abdominal aorta guaranteed satisfactory hemodynamic parameters and ECMO flow , contributing to a rapid improvement of LVEF, allowing a relative fast weaning from ECMO. At 18 months of follow-up, patient is in stable NYHA class II, with an LVEF of $40 \%$ with a good quality of life.

This is an off-label vascular approach for ECMO cannulation. We consider it may be an alternative option, in patients presenting similar conditions, if performed by an expert cardiovascular team.

\section{Conflict of Interest}

None.

\section{References}

1. Ponikowski P, Voors AA, Anker SD, et al. (2016) 2016 ESC Guidelines for the diagnosis and treatment of acute and chronic heart failure. Eur Heart J 37 :2129-2200.

2. Jeffrey Javidfar, Daniel Brodie, Joseph Costa, et al. (2012) Subclavian Artery Cannulation for Venoarterial Extracorporeal Membrane Oxygenation. ASAIO J $58: 494-498$.

3. Chamogeorgakis T, Lima B, Shafii AE, et al. (2013) Outcomes of axillary artery side graft cannulation for extracorporeal membrane oxygenation. J Thorac Cardiovasc Surg 145: 1088-1092.

4. Shotaro Aso, Hiroki Matsui, Kiyohide Fushimi, et al. (2016) The effect of intraaortic balloon pumping under venoarterial extracorporeal membrane oxygenation on mortality of cardiogenic patients: An analysis using a nationwide inpatient database. Crit Care Med 44: 1974-1979. 